\title{
BMJ Open Comparing ivWatch biosensor to standard care to identify extravasation injuries in the paediatric intensive care: a protocol for a randomised controlled trial
}

Craig Antony McBride (i) , ${ }^{1,2,3}$ Sarfaraz Rahiman (1) , ${ }^{4}$ Luregn J Schlapbach (1) , ,,5,6 Jessica A Schults (1) ,2,4,7,8 Tricia M Kleidon, ${ }^{1,2,7}$ Melanie Kennedy, ${ }^{4}$ Rebecca S Paterson, ${ }^{7}$ Joshua Byrnes, ${ }^{2,9}$ Robert S Ware (1) ,2,9 Amanda Judith Ullman (1) 2,6,7

To cite: McBride CA, Rahiman S, Schlapbach LJ, et al. Comparing ivWatch biosensor to standard care to identify extravasation injuries in the paediatric intensive care: a protocol for a randomised controlled trial. BMJ Open 2022;12:e047765. doi:10.1136/ bmjopen-2020-047765

- Prepublication history for this paper is available online. To view these files, please visit the journal online (http://dx.doi org/10.1136/bmjopen-2020047765).

Received 21 February 2021 Accepted 26 January 2022
D) Check for updates

(c) Author(s) (or their employer(s)) 2022. Re-use permitted under CC BY-NC. No commercial re-use. See rights and permissions. Published by BMJ.

For numbered affiliations see end of article.

\section{Correspondence to} Dr Craig Antony McBride; craig.mcbride@health.qld. gov.au

\begin{abstract}
Introduction Peripheral intravenous catheters (PIVCs) frequently fail during therapy administration, resulting in infusates pooling in the surrounding tissue. These extravasation injuries can cause significant pain, tissue destruction and scarring. ivWatch is a biosensor that uses visible and near-infrared light to measure tissue changes surrounding the PIVC and alert clinicians when extravasation may occur. The effectiveness of ivWatch, in comparison to clinical observation, in decreasing injury severity is unknown. The present study aims to investigate whether using ivWatch may potentially detect injury earlier and decrease the severity of PIVC extravasation injuries.
\end{abstract}

Methods and analysis A single centre, parallel group, open-label superiority randomised controlled trial comparing (a) standard care (clinical observation) to (b) ivWatch monitoring in addition to standard care, to decrease the severity of extravasation injuries. 200 children with PIVCs inserted in the distal half of the limb, receiving intermediate-risk to high-risk infusates for $\geq 24$ hours, will be consecutively recruited at a paediatric intensive care unit in Queensland, Australia. The primary outcome is extravasation severity, measured by the Cincinnati Children's Extravasation Harm Scale. Secondary outcomes include severity assessed with three-dimensional camera imaging, extravasation volume, treatment sequelae, the number of PIVCs used and dwell time, quality of life and healthcare costs. The between treatment difference in extravasation severity will be compared using ordinal logistic regression, with the treatment group included as the main effect, and reported with corresponding $95 \%$ Cls. Estimates of value will be presented as net monetary benefits and cost per reduction in extravasation injury severity, both presented with corresponding $95 \%$ credible intervals.

Ethics and dissemination This study received approval from the Children's Health Queensland Hospital and Health Service Human Research Ethics Committee (HREC) (reference number: HREC/20/QCHQ/60867) and the Griffith University HREC (reference number: 2020/310) and will
Strengths and limitations of this study

- First randomised controlled trial evaluating the efficacy of a biosensor using visible and near-infrared light (ivWatch), in comparison to standard care (clinical observation alone), to prevent significant extravasation injuries.

- Allocation will not be concealed to clinical staff since ivWatch is visible and must be managed; however, masked burns physicians and nurses will assign extravasation severity outcomes according to standardised definitions, and data analysis will be performed by the statistician using a masked dataset.

- Secondary outcomes include quality of life and healthcare costs.

The trial is limited to the paediatric intensive care population.

be disseminated via peer-reviewed publications and conference presentations.

Trial registration number ACTRN12620000317998.

\section{INTRODUCTION}

Peripheral intravenous catheters (PIVCs) are one of the most common invasive interventions in paediatric intensive care units (PICUs). While their insertion is vital for treatment administration, injuries and complications associated with PIVC use are an under-recognised patient safety issue. ${ }^{1}$ Previous research has demonstrated PIVC dysfunction, including extravasation, results in the premature failure of up to $40 \%$ of PIVCs in children. ${ }^{2-5}$ Critically ill children are especially vulnerable to extravasation injuries due to their exposure to multiple high-risk drugs, difficulties in vascular access and the common use of sedation, which may 


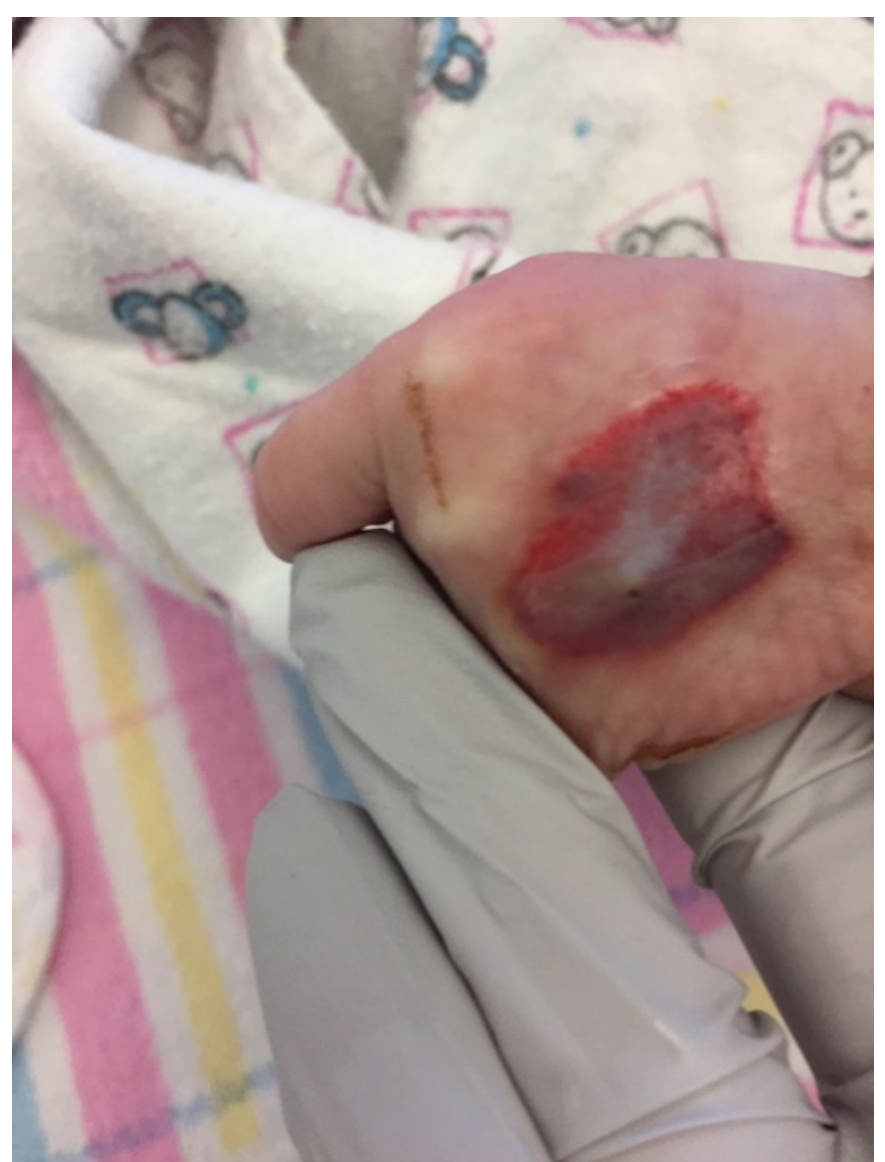

Figure 1 Extravasation injury to the dorsum of the hand 2 days post injury caused by $10 \%$ dextrose and potassium infusion in a 5-week-old infant.

affect timing of detection of extravasation. ${ }^{6-8}$ Extravasation injuries from PIVCs are common, especially in the PICU. They lead to pain and tissue damage at the site of injury. A proportion lead to tissue loss with a subsequent need for reconstructive operations, such as skin grafting and/or later scar revisional surgery. These patients go on to develop lifelong scarring that, in some instances, is also associated with a lifelong functional deficit across a joint or involving underlying muscle groups. In Australia, extravasation injuries caused by inadvertent extravascular delivery of intravenous medication rank among the top five causes of critical incidents in paediatric critical care.

The risk of PIVC-associated extravasation injuries is influenced by several factors: the patient's comorbidities, their current pathology, catheter insertion technique, the infusates and duration of administration. ${ }^{8-10}$ Within the PICU, there is a confluence of these factors, resulting in an increased prevalence and severity of extravasation sequelae, in comparison to other healthcare settings and populations. The larger the volume extravasated, the easier it is for clinicians to identify extravasation injuries. But by then, extensive injury (including necrosis) may have already occurred (figures 1-4).

Early identification and management of extravasation can reduce the severity and sequelae of these healthcareassociated injuries. ${ }^{11}$ Current guidelines recommend

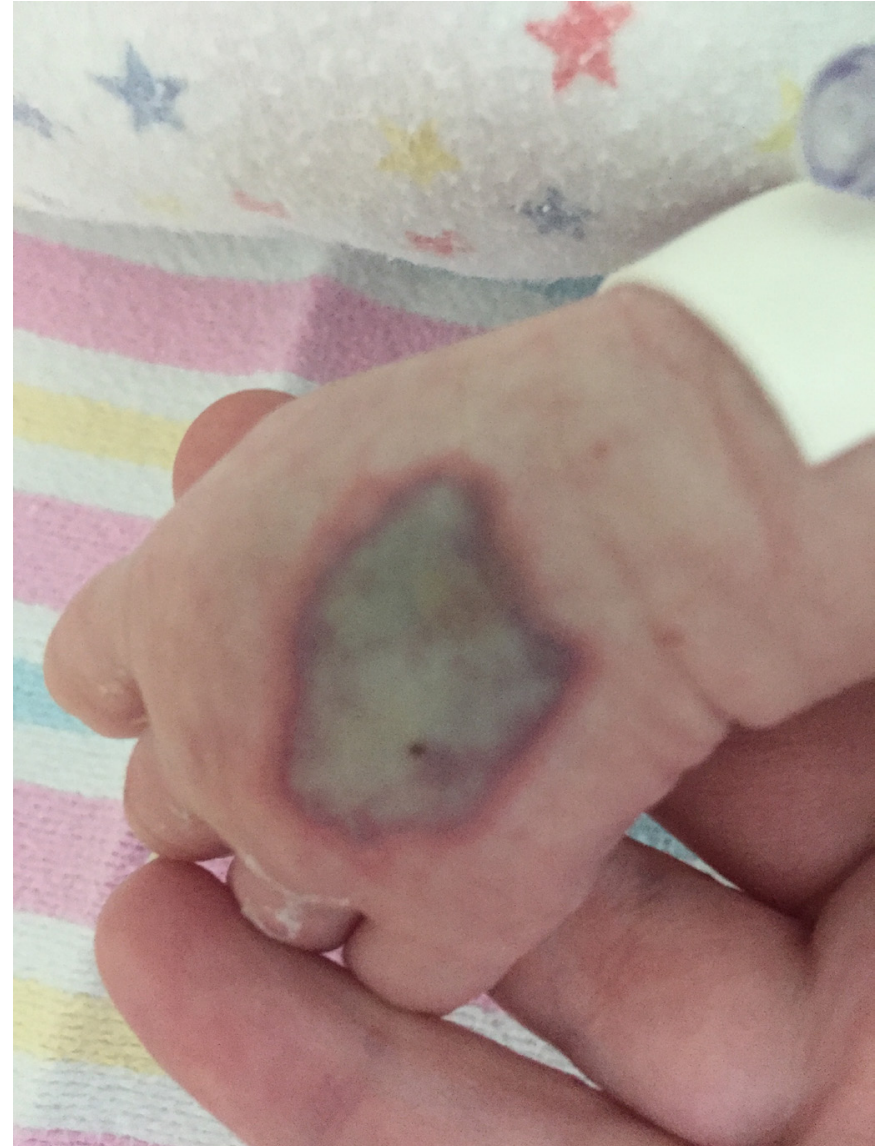

Figure 2 Same patient as figure 1,10 days post injury. Injury treated with nanocrystalline silver dressings. No skin graft required.

hourly assessment of the intravenous site for continuous infusions, and more frequent assessment of high-risk infusions every $5-10 \mathrm{~min}^{12}$ In the busy PICU environment, continuous monitoring of PIVC sites by bedside clinicians is impractical and ineffective. Other patient management tasks, such as airway maintenance or haemodynamic management, may be prioritised over surveillance of an intravenous site. Additionally, the primary early sign of PIVC dysfunction is localised pain, which is extremely difficult to assess in sedated, ventilated or non-verbal children. $^{13} 14$

A potential new technological solution to improve early detection of dysfunctional PIVCs is a biosensor using visible and near-infrared light, known as ivWatch (ivWatch LLC; Newport News, Virginia, USA). ivWatch consists of a patient monitor, a reusable electronic cable and a sterile disposable sensor to secure to the patient's skin adjacent to the PIVC site (figure 5). The ivWatch sensor continuously monitors the optical properties of tissue near the PIVC site, and provides audible and visual notifications when tissue fluid volume changes. While this technology shows significant potential to reduce the sequelae associated with PIVC extravasation, to date there are few data to support its use. A single-site, small, industry sponsored cohort study ( $\mathrm{n}=213 ; 28$ events) at Cincinnati Children's Hospital found ivWatch alerted clinicians to suspected 


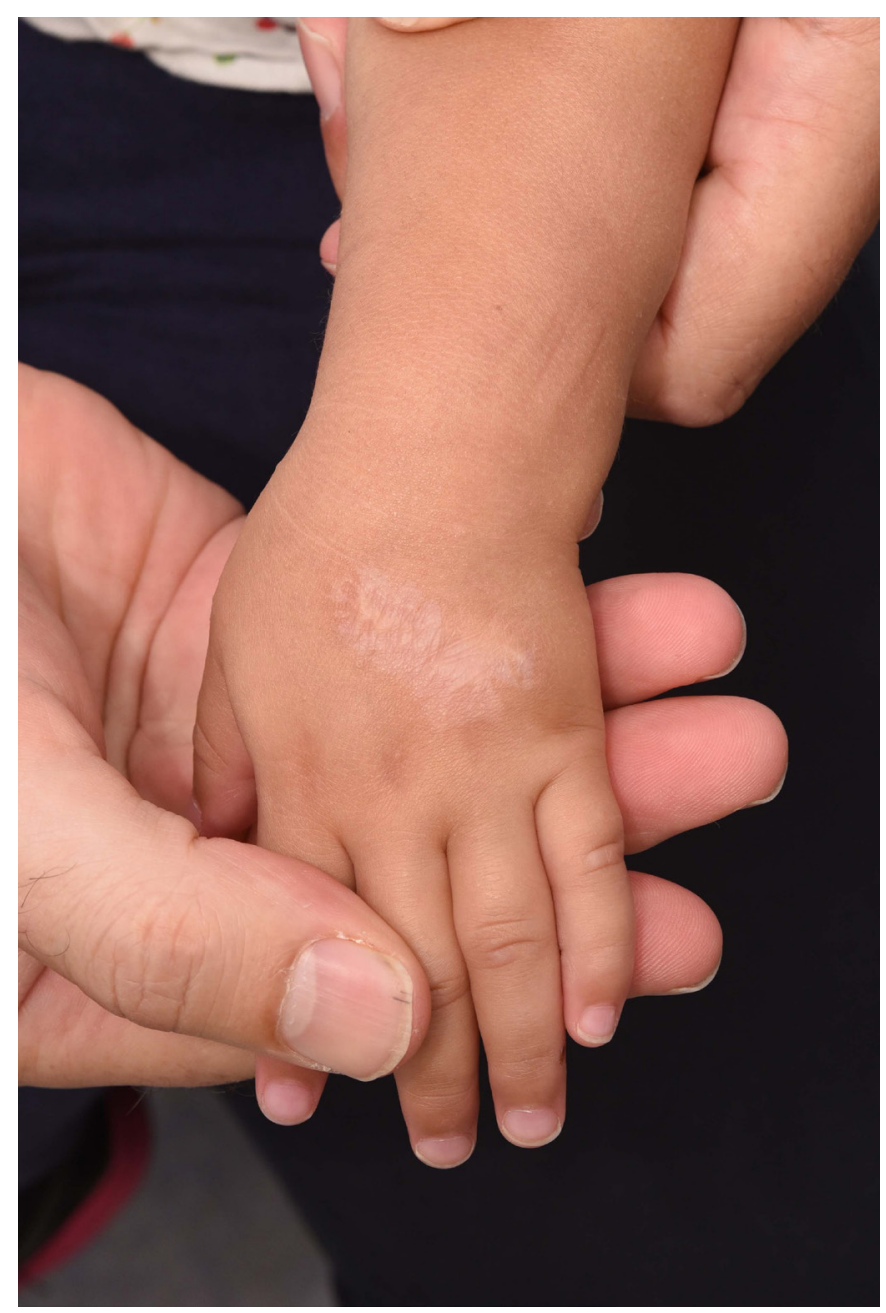

Figure 3 Scar appearance in the same patient 20 months post injury, at conclusion of active scar management therapy.

extravasation in $78 \%$ of cases before clinician detection, improving time to identification by 30 hours (95\% CI 14.8 to 44.8$).{ }^{15}$ Similarly, a recent cohort study in neonates

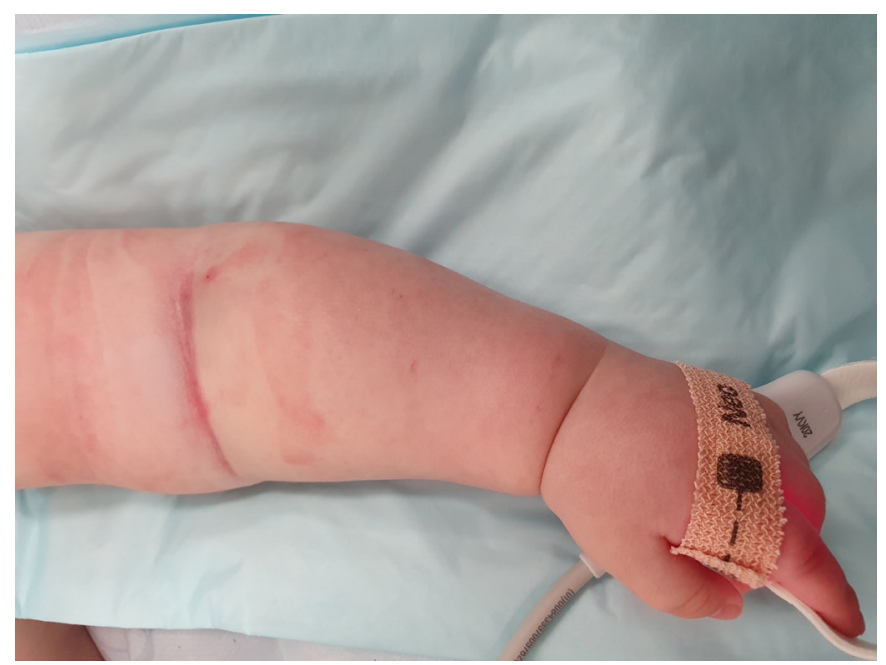

Figure 4 Tissue oedema and blanching from antecubital fossa extravasation of maintenance iv fluid in a 6-month-old infant. $(n=15)$ found ivWatch identified $93.3 \%(n=14)$ of clinically diagnosed, minor infiltration/extravasation events. The manufacturer's white paper states ivWatch has the capability to detect extravasations of as little as $0.22 \mathrm{~mL}$ of infused fluid, with an average detection of an extravasation of $\sim 3 \mathrm{~mL} .{ }^{16}$ In Australia, the Therapeutic Goods Authority has provided product approval, but the clinical effectiveness and value of ivWatch in comparison to current care have not been established.

We aim to conduct an open label randomised controlled trial to evaluate the ivWatch technology in critically ill children treated with intermediate-risk or high-risk intravenous medications.

\section{Study aims}

The overall aim of this study is to decrease the severity of extravasation injuries associated with PIVCs in paediatric intensive care patients. In order to achieve this aim, this project seeks to establish the effectiveness of a biosensor using visible and near-infrared light (ivWatch), in comparison to standard care (clinical observation), in reducing PIVC-associated extravasation injury severity, in the PICU. Therefore, the key objectives of the study are to:

1. Assess the effectiveness of ivWatch, in comparison to standard care, to reduce PIVC-associated extravasation injury severity, in the PICU.

2. Examine the effectiveness of ivWatch, in comparison to standard care, in terms of extravasation volume, quality of life impacts, healthcare costs and their sequelae of extravasation injuries occurring during admission to the PICU.

\section{Hypotheses}

$\mathrm{H}_{0}$ : in children admitted to PICU, PIVCs monitored by ivWatch and standard care in comparison to standard care alone (clinical observation) will not have significantly different PIVC-associated extravasation injury severity.

$\mathrm{H}_{1}$ : in children admitted to PICU, PIVCs monitored by ivWatch in comparison to standard care will have different PIVC-associated extravasation injury severity.

\section{METHODS AND ANALYSIS}

This single-centre, open label randomised controlled superiority trial with two parallel arms is comparing the clinical effectiveness and cost effectiveness of two approaches for extravasation monitoring:

1. Standard care: clinical observation.

2. ivWatch: clinical observation plus the ivWatch monitor, applied within $10-15 \mathrm{~mm}$ of the PIVC cannula tip.

The randomised controlled trial is reported in accordance with the Standard Protocol Items: Recommendations for Interventional Trials 2013 statement for clinical trial protocols. ${ }^{17}$

\section{Study setting and sample}

The trial began recruitment on 7 September 2020 and is expected to accrue participants until April 2022. The 


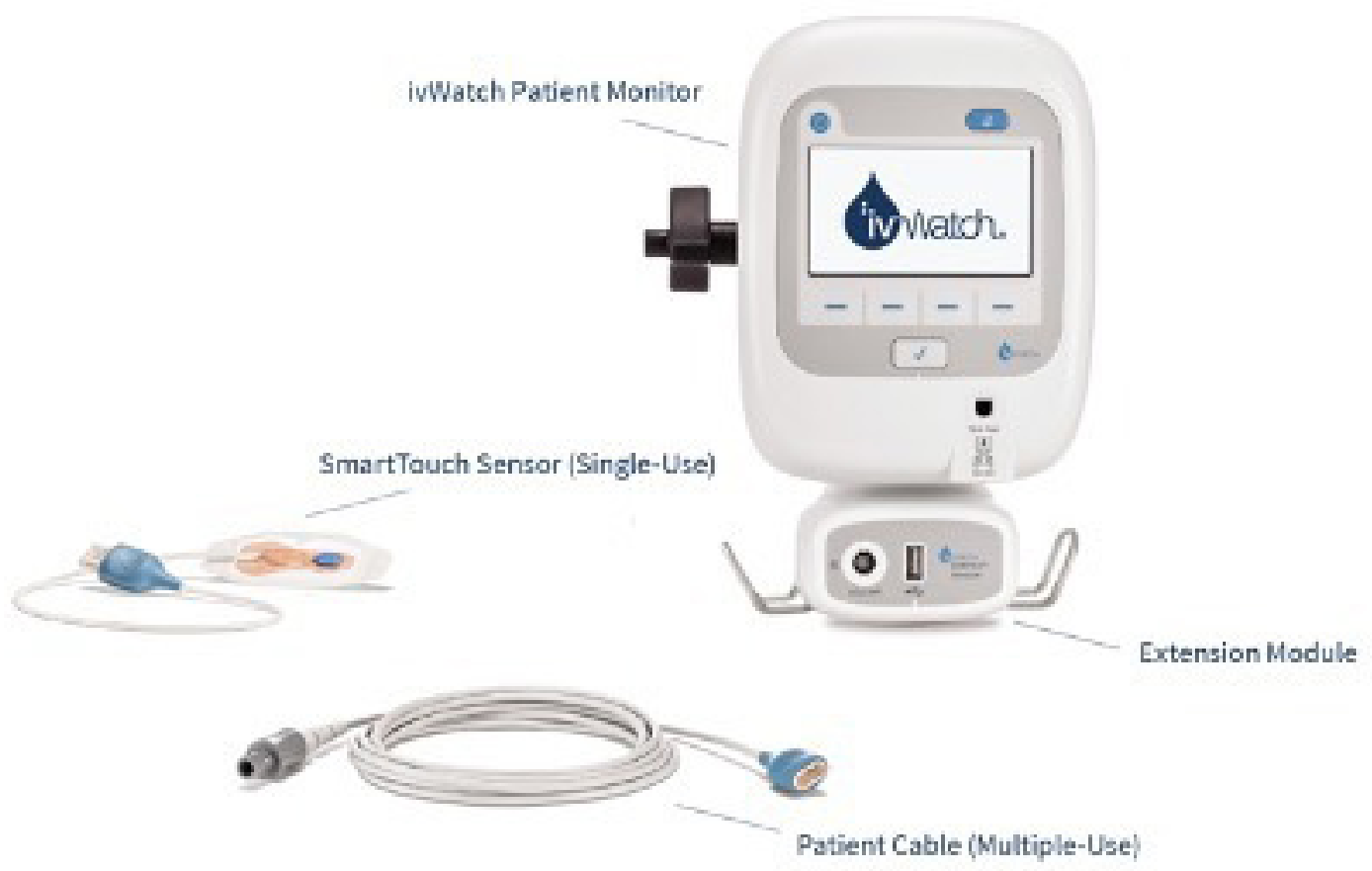

Figure 5 The ivWatch Model 400 Patient Monitoring System (ivWatch LLC; Newport News, Virginia, USA) (image used with permission from the manufacturer).

trial is based at the Queensland Children's Hospital, a tertiary paediatric hospital located in Brisbane, Australia. The PICU has 36 beds, admitting approximately 2000 critically ill children (neonates to 17 years old) annually across medical, surgical and cardiovascular diagnoses. Patients who meet all inclusion and no exclusion criteria (see table 1) are eligible to participate in the study once.

\section{Outcome measures and definitions}

\section{Primary outcome}

The primary outcome is extravasation severity, measured using the 4-point Cincinnati Children's Hospital Medical Centre Extravasation Harm Scale categorised as no, mild, moderate or severe harm. ${ }^{18}$ This Scale combines an assessment of both physical (ie, discolouration, swelling, redness, blisters, function and arterial compromise), infusate ( $\mathrm{pH}$, osmolality, biological activity and volume) and treatment (scarring and surgical management) characteristics (as described in table 2).

\section{Secondary outcomes}

Secondary outcomes include extravasation volume and treatment sequelae, the number of PIVCs used per patient, PIVC dwell time, patient quality of life and associated healthcare costs.

Sample size and study power

Sample size calculations are based on the three-level outcome variable of extravasation severity (no, mild and moderate-severe). ${ }^{18}$ Local and international data report approximately $40 \%$ of PIVCs with intermediate-risk to high-risk infusates result in extravasation injuries in paediatrics under conditions similar to our 'standard care' scenario. ${ }^{235}$ In the control arm, we expect that $70 \%$ of patients will have severity ' 0 ' (no), $25 \%$ will have severity ' 1 ' (mild) and $5 \%$ will have severity $>1$ (moderate-severe). We expect in the ivWatch arm the equivalent probabilities will be $88 \%, 10.4 \%$ and $1.6 \%$. This is equivalent to

\section{Table 1 Inclusion and exclusion criteria}

\begin{tabular}{ll} 
Inclusion criteria & Exclusion criteria \\
\hline PIVC (including a long PIVC) inserted in the distal half of the & Other vascular access devices (including midline \\
limb (ie, up to popliteal or antecubital fossae) & catheters and central venous catheters) \\
PIVC is anticipated to be in situ for $>24$ hours & Previous enrolment in the study \\
Patient anticipated to be admitted to PICU for $>24$ hours & Current 'do not resuscitate' order or receiving palliative \\
Planned administration of $\geq 1$ intermediate-risk to high-risk & care \\
infusates, ${ }^{8}$ antibiotics ${ }^{26}$ or continuous fluids, via the PIVC & Under the protection of the Child and Family Services \\
study & Primary caregivers/legal guardians do not speak English \\
Informed consent from primary caregivers/legal guardians & without the aid of an interpreter
\end{tabular}

PICU, paediatric intensive care unit; PIVC, peripheral intravenous catheter. 
Table 2 Outcomes and measurement instruments

\begin{tabular}{|c|c|}
\hline Outcome(s) & Measurement tool(s) \\
\hline \multicolumn{2}{|l|}{ Primary } \\
\hline Extravasation severity & - Cincinnati Children's Extravasation Harm7 : 4-point scale (0-3, increasing severity) \\
\hline Extravasation volume & $\begin{array}{l}\text { Surface area to limb length ratio } \\
\\
\text { 3D and } 2 \mathrm{D} \text { camera imaging }\end{array}$ \\
\hline PIVCs used and dwell time & $\begin{array}{l}\text { Count of number of PIVCs used per patient } \\
\text { Dwell time of each (in hours) }\end{array}$ \\
\hline Quality of life & Brisbane Burns Scar Impact Profile at 1 month, 3 months and 6 months ${ }^{28}$ \\
\hline Acceptability $^{29-32}$ & $\begin{array}{l}\text { Qualitative and quantitative interviews with parent and clinician surrounding } \\
\text { acceptability with study intervention and control }\end{array}$ \\
\hline
\end{tabular}

2D, two-dimensional; 3D, three-dimensional; PICU, paediatric intensive care unit; PIVC, peripheral intravenous catheter.

specifying a proportional OR of 0.32 . With $\alpha=0.05$ and power $=90 \%$, we are required to record outcome data on 96 participants in each group to detect a between-group difference of this size or greater. ${ }^{19}$ The proposed decrease in severity is conservative compared with the $78 \%$ relative decrease observed in previous cohort studies. ${ }^{1520}$

\section{Recruitment, randomisation, allocation concealment and masking}

Clinical research nurses (ReNs) based in the PICU are screening patients for inclusion in the study, and approaching and gaining informed consent from patients or parents after consultation with the PICU team. ReNs are also randomising patients, educating clinical staff, monitoring protocol compliance and collecting 7-day and 28-day post-trial outcome data. Each patient is randomised using a central web-based service (Griffith University) in a 1:1 ratio. Block sizes are either 8 or 10 (size randomly selected).

Masking clinical staff and patients and their parents to treatment allocation is not possible, as PIVCs accompanied by the ivWatch device are visibly different to those without. However, assessment and treatment of an extravasation injury are measured using objective, validated measures and routinely assigned to clinical staff who are not study investigators, facilitating partial masking for primary and secondary outcomes. In particular, assessment and treatment of the injury is occurring following referral of the patient, allowing for the removal of the ivWatch device (where used) in order to ensure masking. The biostatistician will be masked to allocation when provided with the final dataset.

\section{Insertion and management of PIVC}

If allocated, the ivWatch sensor is being applied adjacent to the catheter tip site, in accordance with manufacturer's instruction manual. All other aspects of PIVC insertion, management and removal are standardised in accordance with Queensland Health clinical practice guidelines. ${ }^{21}$ Insertion, maintenance and removal are performed by the usual PICU interdisciplinary staff. There are no new requirements regarding this that are particular to this study, as our intention is a pragmatic trial. Where patients are randomised to the ivWatch arm of the trial, a corner of the PIVC dressing may be lifted to place the sensor appropriately on the skin.

\section{Data collection}

Quantitative data are being collected by the clinical ReNs from the electronic medical record, and entered into a dedicated secure, online database (Research Electronic Data Capture (REDCap)). ${ }^{22}$ Once enrolled in the study, participants are followed up daily by ReNs until the PIVC is removed or the patient is discharged from PICU, and again at 7 days and 28 days. A timeline of the study participation is summarised in table 3. Patients discharged from the hospital service are having follow-up data collected by phone, a method for which we have previously demonstrated reliability. ${ }^{23}$ The study manager is undertaking quality checks and monitoring of $100 \%$ source data verification for: all data for the first patient, all consent forms, all primary endpoints, as well as a random $5 \%$ of other data for all patients.

At enrolment: ReNs collect consent forms, and demographic and clinical data, to enable a description of the 
Table 3 Schedule of enrolment, interventions and assessments

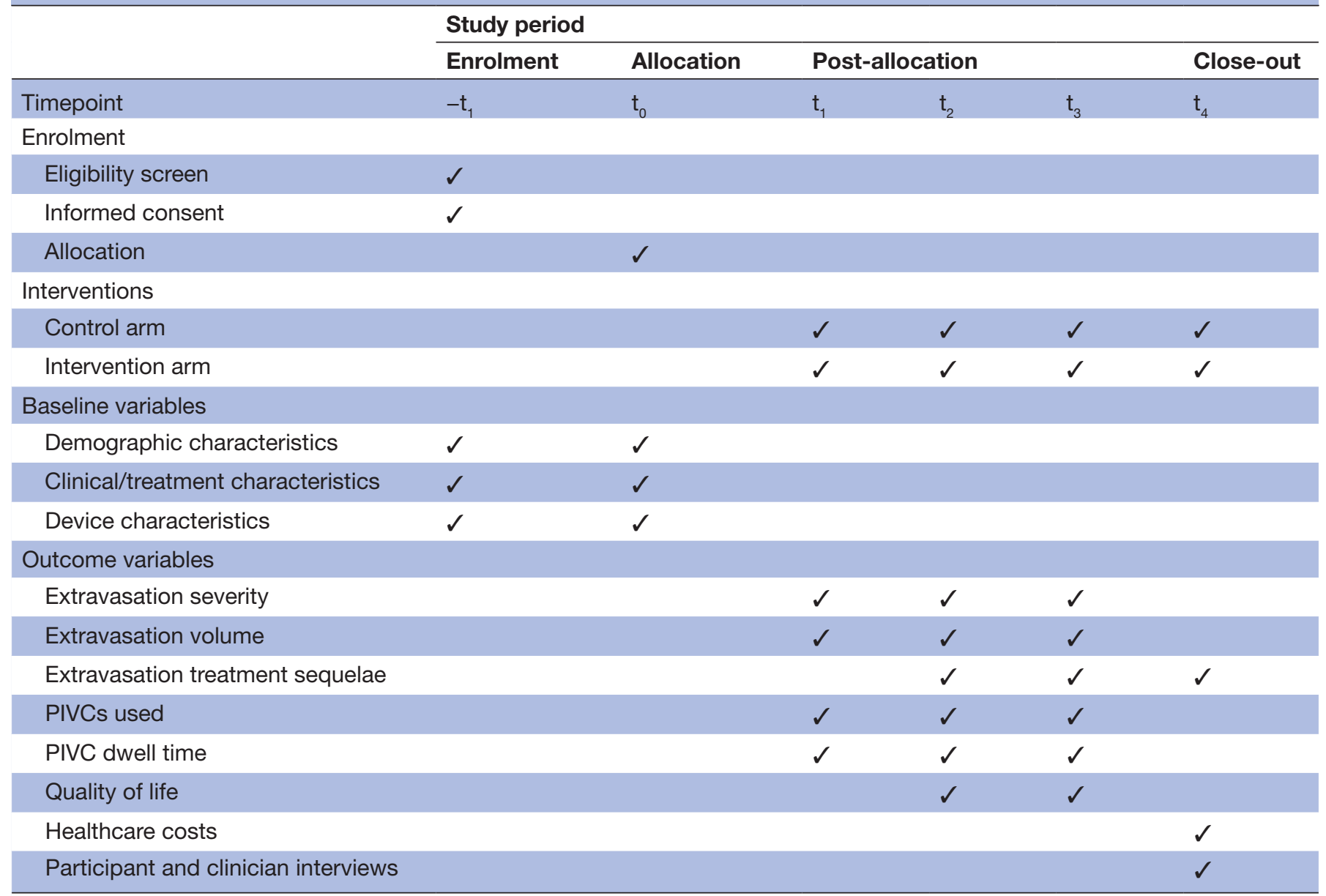

Control arm: standard care; intervention arm: standard care +ivWatch; $-\mathrm{t}_{1}$ : admitted to PICU requiring a PIVC; $\mathrm{t}_{0}$ : enrolled in the study and randomised to either the intervention or control arm of the study; $t_{1}$ : daily checks of PIVC while inserted and patient admitted to PICU; $t_{2}: 7-$ day follow-up following PIVC removal or discharge from PICU; $t_{3}$ : 28-day follow-up following PIVC removal or discharge from PICU; and $t_{4}$ : end of the study.

PICU, paediatric intensive care unit; PIVC, peripheral intravenous catheter.

study participants (eg, age, primary diagnosis and length of stay) and device characteristics (eg, insertion location and gauge).

Daily: ReNs perform checks on enrolled patients to assess for primary and secondary outcomes; current use; and any clinical, PIVC or treatment factors that have changed since recruitment. On signs of extravasation, they refer any extravasation presentations to the paediatric burns inpatient medical and nursing teams.

Within 7 days and up to 28 days following PIVC removal or discharge from PICU: ReNs contact participants to collect clinical data related to treatment sequelae and health-related quality of life data. In addition, ReNs are conducting semi-structured interviews with patients and/ or parents, and clinicians to collect qualitative and quantitative data on acceptability of the ivWatch device (see table 4).

Extravasation data collection: after referral to the paediatric burns inpatient medical and nursing teams, allocation masked data collection surrounded the nature and severity of the extravasation injury, and treatment and outcomes are collected and entered into the same REDCap database.

\section{Data analysis}

Patient characteristics will be summarised descriptively. Continuous data will be summarised as mean (SD) or median (25th-75th percentile) as appropriate. The primary outcome of extravasation severity will be investigated using ordinal logistic regression with treatment group included as the main effect. Secondary outcomes measured on an interval scale will be assessed using linear regression models, while binary outcomes will be assessed using logistic regression models and count outcomes will be assessed using Poisson regression models. In all regression models, treatment group will be included as the main effect, and the baseline value of the outcome will be included as a covariable if appropriate. Effect estimates will be reported with $95 \%$ CIs. Analyses will be conducted on an intention-to-treat basis. The cause of any missing data will be assessed, and sensitivity analyses to investigate their potential impact will be undertaken using multiple 


\begin{tabular}{ll}
\hline Table 4 Qualitative interview questions \\
\hline Clinicians & Families/caregivers \\
\hline What was your & How did you find ivWatch? \\
experience with ivWatch? & \\
Did the presence of & Have you ever had an \\
ivWatch influence your & experience with an \\
ability to assess the & extravasation injury with a \\
PIVC, insertion site or & PIVC? \\
surrounding area? & \\
Do you have any & Was the presence of \\
comments on the ivWatch & ivWatch acceptable to \\
monitoring equipment? & you? (NRS: 1-10) \\
Do you have any further & \\
comments regarding & \\
ivWatch? & \\
Was the presence of \\
ivWatch acceptable to \\
you? (NRS: 1-10)
\end{tabular}

NRS, Numerical Rating Scale; PIVC, peripheral intravenous catheter.

imputation techniques if appropriate. A per-protocol analysis will assess the effect of protocol violations (ie, ivWatch not applied). Statistical tests will be considered significant at the $95 \%$ level $(\mathrm{p}<0.05$, two-tailed).

For the economic assessment, the primary outcome is the incremental net monetary benefit of ivWatch compared with usual care and a secondary outcome of cost per reduction in extravasation injury severity. Primary analysis will be from the perspective of the healthcare sector. Healthcare costs will be derived as the sum product of healthcare resources and their respective price, including labour time, consumables, medication and length of stay. In addition, the total cost per ivWatch, including associated training, maintenance and other costs, will be reported. Estimates of the cost of healthcare utilisation associated with treatment of injury sequelae will be based on resource measurement collected within the trial. Subsequent reconstructive operations, such as skin grafting and/or scar revisional surgery, will be costed based on current treatment protocols and existing hospital cost estimates. Estimates of the 95\% credible interval using non-parametric bootstrapping will be presented to characterise uncertainty in the economic outcomes.

Qualitative interviews will be thematically analysed using standard techniques. ${ }^{24}$

\section{Patient and public involvement}

Feedback regarding the acceptability of the ivWatch device is being sought from patients and their families, and clinicians in qualitative interviews (table 4). These will be thematically analysed and published in a peerreviewed journal alongside the quantitative trial data. The final results of the study will be distributed to study participants, via a plain language summary and accompanying peer-reviewed articles, to their provided email address.

\section{Ethics and dissemination}

This trial is registered with the Australian and New Zealand Clinical Trial Registry. Ethical approval for the trial has been obtained from Children's Health Queensland Hospital and Health Service Human Research Ethics Committee (HREC) (reference number: HREC/20/ QCHQ/60867) and Griffith University HREC (reference number: 2020/310). This study is being performed in accordance with the ethical principles of the Declaration of Helsinki's Note for Guidance on Good Clinical Practice (CPMP/ICH-135/95) and with Australian Government NHMRC National Statement on Ethical Conduct in Research Involving Humans. ${ }^{25}$

A data safety management board (DSMB) has been established to review the safety of ivWatch and to monitor the progress of the trial. The DSMB is compromised of three committee members; appointed by the research team and based on their clinical experience with PIVCs and extravasation injuries. The DSMB includes a statistician. The DSMB will convene an interim meeting 3 months after the trial commences, or at the recruitment of 100 patients (whichever occurs first). Committee members will be unmasked to key interim trial results, in particular severe extravasation injuries and PIVC replacements, per group.

No new adverse events are expected as the ivWatch technology is a surface, rather than an implanted, device and no adverse events have been previously noted in relation to its use. ${ }^{1520}$ Any serious adverse events and protocol modifications will be reported to the HRECs. If important protocol modifications are required (eg, changes to eligibility criteria, outcomes or analyses), CAM will update all investigators, HRECs, information and consent forms, the trial registry and BMJ Open. Clinical trial insurance is held by the university. Prospective written and informed consent will be sought from the patient (where appropriate) or the patient's parent or guardian by the ReN prior to enrolment in the study. Consent can be withdrawn. Withdrawal data will be collected and included in the Consolidated Standards of Reporting Trials diagram. Participants will not be identified by name, and confidentiality of all medical record information will be preserved. All participants details will be entered in coded format and the confidentiality of the participant will be maintained unless disclosure is required by law.

The trial will be written by the investigators and will be submitted to a peer-reviewed medical journal, preferably under an open access format to permit free access to the published study. De-identified raw data will be stored within a data repository held at Griffith University. 


\section{Trial status}

The trial commenced on the 7 September 2020. A total of 39 patients had been recruited at time of manuscript submission, with a planned 19-month recruitment period.

\section{Data statement}

As the trial is ongoing, data generated and/or analysed are not yet publicly available. On completion of the trial, data sets will be available from the chief investigator $(\mathrm{CAM})$ on reasonable request and following review of such a request by the original ethics committees.

\section{DISCUSSION}

Extravasation injuries are a common and preventable injury. Failure to detect extravasation early leads to multiple adverse events, including significant scarring. Innovations in visible and near-infrared technologies, such as ivWatch, may led to earlier detection of potentially harmful events and consequently reduce preventable harm to patients and reduce clinician burden.

\section{Author affiliations}

${ }^{1}$ Surgical Team: Infants, Toddlers, Children (STITCh), Queensland Children's Hospital, South Brisbane, Queensland, Australia

${ }^{2}$ Alliance for Vascular Access Teaching and Research Group, Griffith University, Nathan, Queensland, Australia

${ }^{3}$ Pegg Leditschke Children's Burns Centre, Children's Health Queensland, South Brisbane, Queensland, Australia

${ }^{4}$ Paediatric Intensive Care Unit, Queensland Children's Hospital, South Brisbane, Queensland, Australia

${ }^{5}$ Department of Intensive Care Medicine and Neonatology, Children's Research Center, University Children's Hospital Zürich, Zurich, Switzerland

${ }^{6}$ Child Health Research Centre, The University of Queensland, South Brisbane, Queensland, Australia

${ }^{7}$ School of Nursing, Midwifery and Social Work, The University of Queensland, St Lucia, Queensland, Australia

${ }^{8}$ Herston Infectious Disease Institute, Metro North Health, Herston, Queensland, Australia

${ }^{9}$ Centre for Applied Health Economics, Menzies Health Institute, Griffith University, Southport, Queensland, Australia

Twitter Craig Antony McBride @PaedSurg, Jessica A Schults @jess_schults and Amanda Judith UIIIman @a_ullman

Acknowledgements We would like to acknowledge the contributions made by the patients and families admitted to paediatric intensive care unit (PICU) and to the staff of PICU.

Contributors CAM and AJU conceived the study. CAM, AJU and JB designed the economic evaluation. CAM, SR, LJS, JAS, TK, RSP, JB, RSW and AJU designed the protocol. CAM, LJS, JAS, TK, MK, JB, RSW and AJU secured funding. CAM, SR, LJS, JAS, TK, MK, RSP, JB, RSW and AJU prepared and approved the final version of the manuscript.

Funding This work was supported by the Children's Hospital Foundation (RPC0822020). Financial support for publication was provided by the Centre for Children's Burn and Trauma Research. Consumables were provided by ivWatch, LLC. No commercial entity had any role in the conception, design or funding of this study. No commercial entity will have any role in the collection, management, analysis or interpretation of data; writing of the report; or the decision to submit the report for publication. Authority for all of these activities rests with the above authors.

Competing interests CAM reports travel and accommodation expenses from Roche and Smith \& Nephew (unrelated to the current project), and a research grant provided to the Pegg Leditschke Children's Burns Centre from Abigo Medical AB (unrelated to the current project). JAS reports investigator-initiated research grants provided to Griffith University from BD-Bard (unrelated to the current project). TK reports investigator-initiated research grants and speaker fees provided to Griffith University from 3M Medical, Angiodynamics, Baxter, BD-Bard and Medical Specialties Australia (unrelated to the current project). JB reports investigatorinitiated research grants provided to Griffith University from Becton Dickinson and Navi Technologies (unrelated to current project). AJU reports investigator-initiated research grants and speaker fees provided to Griffith University from 3M Medical, Becton Dickinson, Cardinal Health and Navi Technologies (unrelated to the current project). The other authors have no conflicts of interest to declare.

Patient and public involvement Patients and/or the public were involved in the design, or conduct, or reporting, or dissemination plans of this research. Refer to the Methods section for further details.

Patient consent for publication Consent obtained from parent(s)/guardian(s).

Provenance and peer review Not commissioned; externally peer reviewed.

Open access This is an open access article distributed in accordance with the Creative Commons Attribution Non Commercial (CC BY-NC 4.0) license, which permits others to distribute, remix, adapt, build upon this work non-commercially, and license their derivative works on different terms, provided the original work is properly cited, appropriate credit is given, any changes made indicated, and the use is non-commercial. See: http://creativecommons.org/licenses/by-nc/4.0/.

ORCID iDs

Craig Antony McBride http://orcid.org/0000-0001-8377-1748

Sarfaraz Rahiman http://orcid.org/0000-0002-7716-2919

Luregn J Schlapbach http://orcid.org/0000-0003-2281-2598

Jessica A Schults http://orcid.org/0000-0002-5406-9519

Robert S Ware http://orcid.org/0000-0002-6129-6736

Amanda Judith Ullman http://orcid.org/0000-0001-8860-5319

\section{REFERENCES}

1 Rickard CM, Marsh NM. Web Exclusives. Annals for Hospitalists Inpatient Notes - The Other Catheter-the Mighty Peripheral IV. Ann Intern Med 2017;167:HO2-3.

2 Kleidon TM, Cattanach P, Mihala G, et al. Implementation of a paediatric peripheral intravenous catheter care bundle: a quality improvement initiative. J Paediatr Child Health 2019;55:1214-23.

3 Legemaat M, Carr PJ, van Rens RM, et al. Peripheral intravenous cannulation: complication rates in the neonatal population: a multicenter observational study. J Vasc Access 2016;17:360-5.

4 Malyon L, Ullman AJ, Phillips N, et al. Peripheral intravenous catheter duration and failure in paediatric acute care: a prospective cohort study. Emerg Med Australas 2014;26:602-8.

5 Özalp Gerçeker G, Kahraman A, Yardimci F, et al. Infiltration and extravasation in pediatric patients: a prevalence study in a children's Hospital. J Vasc Access 2018;19:266-71.

6 Amjad I, Murphy T, Nylander-Housholder L, et al. A new approach to management of intravenous infiltration in pediatric patients: pathophysiology, classification, and treatment. J Infus Nurs 2011;34:242-9.

7 Laudenbach N, Braun CA, Klaverkamp L, et al. Peripheral i.v. stabilization and the rate of complications in children: an exploratory study. J Pediatr Nurs 2014;29:348-53.

8 Clark E, Giambra BK, Hingl J, et al. Reducing risk of harm from extravasation: a 3-tiered evidence-based list of pediatric peripheral intravenous infusates. J Infus Nurs 2013;36:37-45.

9 Patregnani JT, Sochet AA, Klugman D. Short-Term peripheral vasoactive infusions in pediatrics: where is the harm? Pediatr Crit Care Med 2017;18:e378-81.

10 Rupp JD, Ferre RM, Boyd JS, et al. Extravasation risk using ultrasound-guided peripheral intravenous catheters for computed tomography contrast administration. Acad Emerg Med 2016;23:918-21.

11 Park SM, Jeong IS, Kim KL, et al. The effect of intravenous infiltration management program for hospitalized children. J Pediatr Nurs 2016;31:172-8.

12 Gorski LA, Hallock D, Kuehn SC, et al. Recommendations for frequency of assessment of the short peripheral catheter site. J Infus Nurs 2012;35:290-2.

13 Rickard CM, Ray-Barruel G. Peripheral intravenous catheter assessment: beyond phlebitis. Lancet Haematol 2017;4:e402-3.

14 Maaskant J, Raymakers-Janssen P, Veldhoen E, et al. The Clinimetric properties of the comfort scale: a systematic review. Eur J Pain 2016;20:1587-611. 
15 Doellman D, Rineair S. The use of optical detection for continuous monitoring of pediatric IV sites. Journal of the Association for Vascular Access 2019;24:44-7.

16 Bonnema GT, Schears G, Naramore W. ivWatch model 400: device validation for Non-Infiltrated tissue ClinicalTrials.gov, 2014.

17 Chan A-W, Tetzlaff JM, Altman DG, et al. Spirit 2013 statement: defining standard protocol items for clinical trials. Ann Intern Med 2013;158:200-7.

18 Royal Children's Hospital Melbourne. Clincial guidelines: extravasation injury management. Melbourne, Australia, 2020.

19 Whitehead J. Sample size calculations for ordered categorical data. Stat Med 1993;12:2257-71.

20 van Rens M, Hugill K, Velez Francia A. A new approach for early recognition of peripheral intravenous (Piv) infiltration: a pilot appraisal of a sensor technology in a neonatal population. Vascular Access 2019;5:38-41.

21 Children's Health Queensland Hospital and Services. Venous Access Device (VAD)-Insertion and management of peripheral and central venous access devices. Brisbane: Queensland Government, 2018.

22 Harris PA, Taylor R, Thielke R, et al. Research electronic data capture (REDCap)--a metadata-driven methodology and workflow process for providing translational research informatics support. J Biomed Inform 2009;42:377-81.

23 Webster J, Northfield S, Marsh N. Excellent inter-rater agreement of peripherally inserted central catheter site condition between nurses and patients: PICC-SAT. J Vasc Access 2018;19.

24 Vaismoradi $\mathrm{M}$, Turunen $\mathrm{H}$, Bondas T. Content analysis and thematic analysis: implications for conducting a qualitative descriptive study. Nurs Health Sci 2013;15:398-405.
25 National Health and Medical Research Council. National statement on ethical conduct in human research: 2007 (updated 2018): Commonw, 2018. Aust Canberra. Available: https://www.nhmrc. gov.au/about-us/publications/national-statement-ethical-conducthuman-research-2007-updated-2018

26 Salgueiro-Oliveira A, Parreira P, Veiga P. Incidence of phlebitis in patients with peripheral intravenous catheters: the influence of some risk factors. Australian Journal of Advanced Nursing, The 2012;30:32.

27 Infusion Nurses Society. Infusion therapy standards of practice. J Infus Nurs 2016;39 https://source.yiboshi.com/20170417/ 1492425631944540325.pdf

28 Tyack Z, Ziviani J, Kimble R, et al. Measuring the impact of burn scarring on health-related quality of life: development and preliminary content validation of the Brisbane burn scar impact profile (BBSIP) for children and adults. Burns 2015;41:1405-19.

29 Sitges-Serra A, Liñares J, Pérez JL, et al. A randomized trial on the effect of tubing changes on hub contamination and catheter sepsis during parenteral nutrition. JPEN J Parenter Enteral Nutr 1985;9:322-5.

30 Anaissie E, Samonis G, Kontoyiannis D, et al. Role of catheter colonization and infrequent hematogenous seeding in catheterrelated infections. Eur J Clin Microbiol Infect Dis 1995;14:134-7.

31 Reynolds H, Taraporewalla K, Tower M, et al. Novel technologies can provide effective dressing and securement for peripheral arterial catheters: a pilot randomised controlled trial in the operating theatre and the intensive care unit. Aust Crit Care 2015;28:140-8.

32 Collignon PJ, Munro R. Laboratory diagnosis of intravascular catheter associated sepsis. Eur J Clin Microbiol Infect Dis 1989;8:807-14. 